\title{
Protection against the Effects of the Asynchronous Operation of Synchronous Motors Based on the Principle of Comparison of the Machine Power Factor
}

\author{
Petr Gric \\ PEG s.r.o., Praha,p.gric@peg.cz
}

\begin{abstract}
This paper deals with the possibility of using the principle of comparison of the operational power factor for evaluation of the asynchronous operation of synchronous motors. This way of definition of the asynchronous operation is possible to use for motors protection and other protective systems of drives with synchronous motors.
\end{abstract}

Keywords - synchronous motors, asynchronous operation, power factor evaluation.

\section{INTRODUCTION}

Synchronous machines are used in many applications thanks to the defined rotor speed of synchronous machines to stator frequency and the possibility of control the reactive part of energy. Asynchronous operation is a nonstandard state when the rotor of a synchronous machine is not rotating synchronously with the rotating field of the stator. This state can occur when the machine is overloaded, the rotor is under exited, due to the fluctuation of power supply voltage or combination of these bearings. Long term asynchronous operation is an inadmissible state. Perhaps we could say an emergency state of synchronous machines.

\section{ASYNCHRONOUS OPERATION}

Start-up winding, so-called damper (amortisseur) winding, is the most strained part during asynchronous operation. Machine, especially rotor winding, can be permanently damaged during long term asynchronous operation. For example, the rotor rod can be unsoldered, or the couplings (screws) of the damper winding could be overheated and destroyed. These parts can consequently damage the stator winding of the synchronous machine.

The time that the synchronous motors can operate in asynchronous mode differs according to the nature of the load and construction of the machine. The rotors of the synchronous machines are designed for asynchronous startup, so they are dimensioned for short term asynchronous operation. I have encountered a case where the synchronous drive of a mining ventilator with a power rating of $3250 \mathrm{kVA}$ was working in an asynchronous mode approximately for 8 hours because of an excitation problem. The drive could not be shut down because of the safety of the miners. Only the power was reduced to approximately one-third of $\mathrm{P}_{\mathrm{N}}$. After the drive was shut down, the revision of the rotor and stator winding was made, but no adverse effect of the long-term operation was discovered.

On the other side, the synchronous motor of $4000 \mathrm{~kW}$ that drives the piston compressor was destroyed after $30 \mathrm{~s}$ of asynchronous operation at $0.75 \mathrm{P}_{\mathrm{n}}$. The cause was a meltdown of a damper coupling and, consequently, the destruction of the stator winding.

Synchronous machines need to be protected against the consequences of the asynchronous operation due to the possibility of a crash.

The asynchronous operation of synchronous motor occurs, of course, during start-up when the motor is connected directly or indirectly through a start-up reactor to the power grid. After start-up to almost synchronous rotations (and short-circuit of start-up reactor), the excitation is connected, and the rotor is pulled to synchronous rotations. This procedure is considered for normal operation and the motor is designed for it. Rotor winding cannot be an open circuit during start-up. It must be short-circuited either directly or through a resistor or some other device that will limit the voltage inducted to the excitation winding during start-up. If the excitation is constant and the load is getting higher, than the machine goes to the inductive (under-excited) part of the operation area. With further increasing of the load (or decreasing of the excitation or a stator voltage), the inductive power factor is getting lower to the limit of stable operation of the machine. The limit is at $\cos \varphi$ lower than 0.5 ind. at machines with salient poles, and $\cos \varphi$ is less than one at machines with a cylindrical rotor. Copy of capability diagram of stability for synchronous generator with a cylindrical rotor with a power rating of 31 MVA is shown in Fig. 1. The operational limit can be seen in the inductive part under $\cos \varphi=0.99$, which practically excludes operation in under-excitation state with a nominal load. The operation in the over-excitation part is not dangerous for a synchronous machine, and it is only limited by the value of phase stator current that cannot be higher than $\mathrm{I}_{\mathrm{N}}$. The value of the phase current is equal to the vector product of the real and imaginary part of the phase current.

$$
\text { If }=|\overrightarrow{I a}+\overrightarrow{I r}|
$$

If - phase stator current of synchronous motor

$\overrightarrow{I a}$ - the real part of phase current

$\overrightarrow{I r}$ - the imaginary part of phase current 


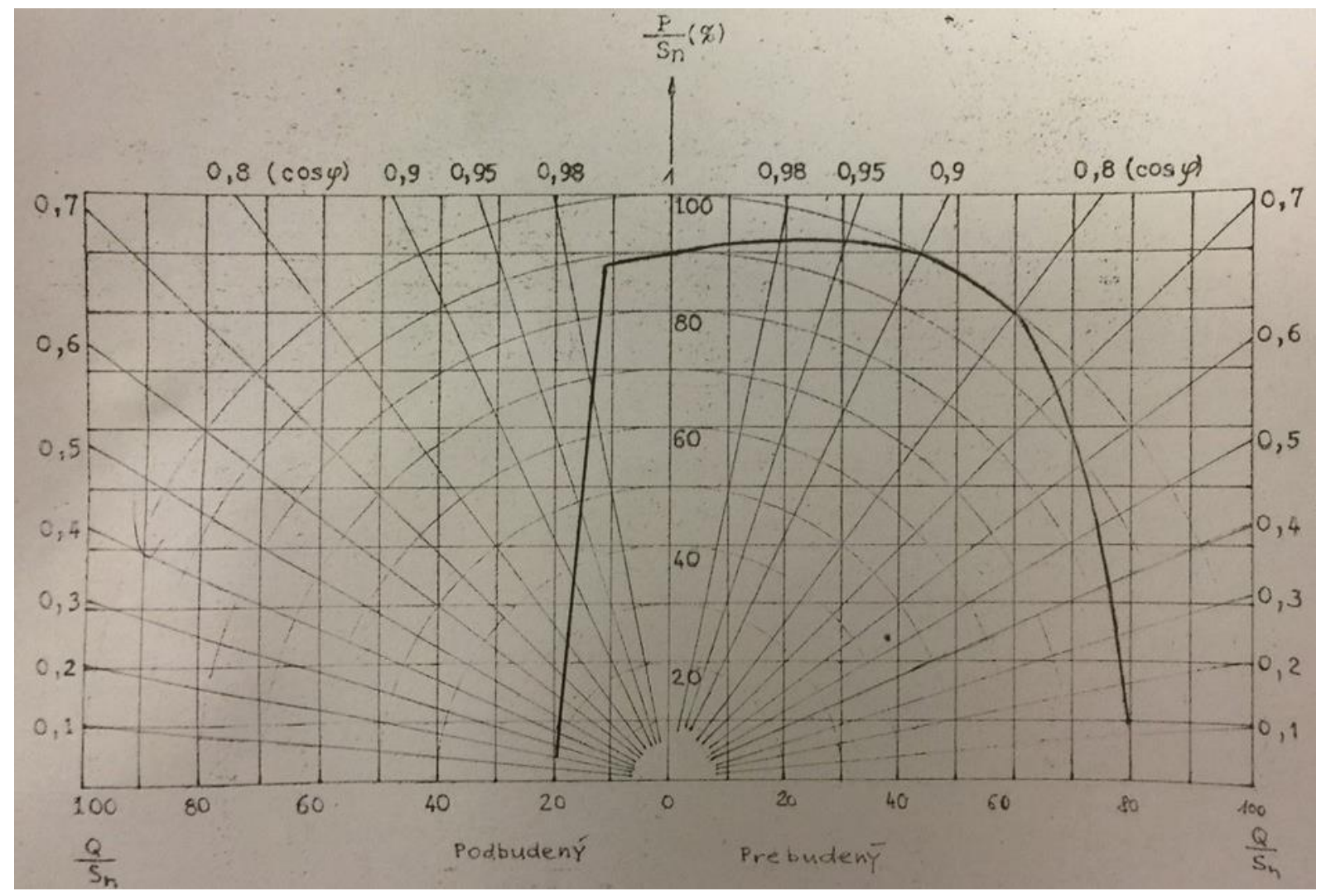

Fig. 1. Capability diagram of synchronous generator 31 MVA.

At the listed values of $\cos \varphi$, the machine falls out of synchronism and rotor slip up for a pole span with regard to the rotating magnetic field of the stator. This phenomenon cyclically repeats, and asynchronous operation occurs unless there is not a rise of excitation current (e.g., due to the influence of under-excitation limit of excitation source) or the power of the load is not lowered.

This process is described in Fig. 2 and Fig. 3.

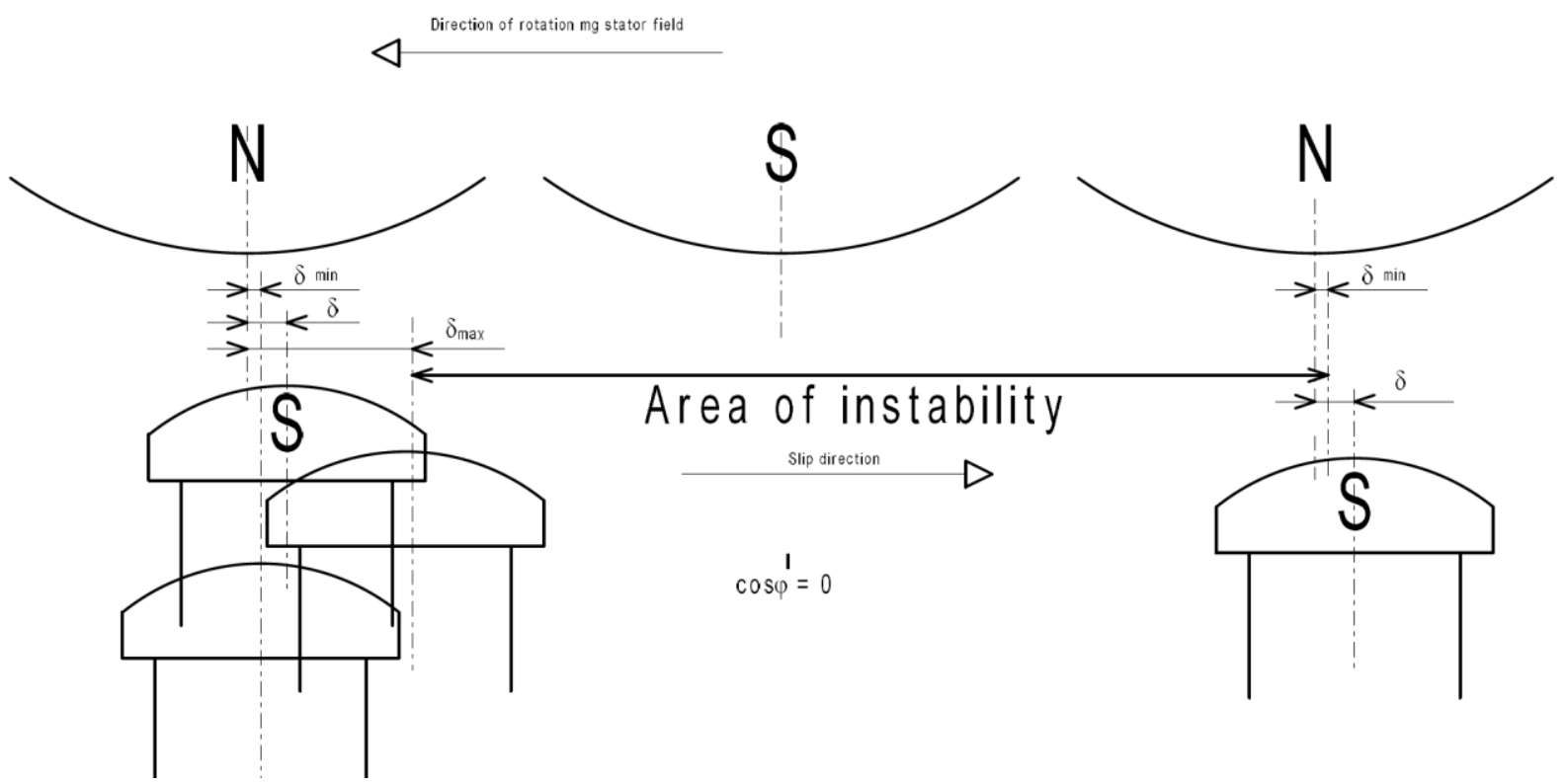

Fig. 2. Slip up for a pole span with regard to torque angle of the machine. 


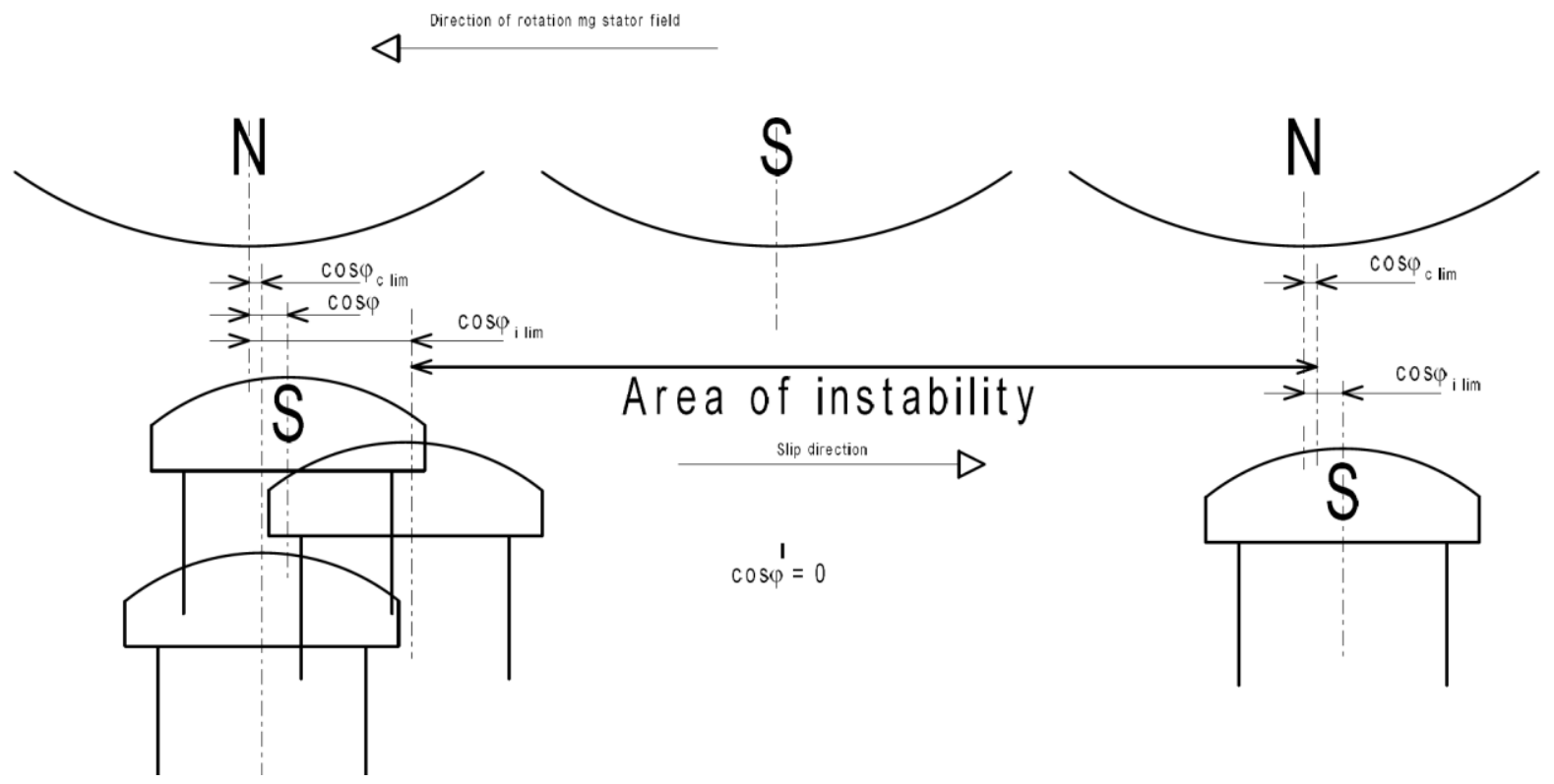

Fig. 3. Slip up for a pole span with regard to power factor of the machine

Torque angles for each state of the machine (motor operation) are shown in Fig. 2.

$\delta \mathrm{min}$ - torque angle for $\mathrm{P}=0$, Ibn and Un (idle operation, nominal excitation current), torque angle minimal

$\delta$ - operational torque angle, motor works at nominal load, operational value of excitation current and nominal stator voltage

Smax - maximal torque angle that corresponds to stability limit, when this angle is exceeded the magnetic bond is torn and the rotor slips up for a pole span

When the magnetic bond between the rotor and stator magnetic field is torn, the slip up occurs. It goes through a stage of instability (magnetic poles with the same polarity are repelled) back to the working area. If there is no change either torque (the load torque does not change) or electrical (an increase of stator voltage, an increase of excitation current), the rotor will not hold in the working area and the slip-up cycle repeats itself.

Torque angle is proportional to the load, stator voltage and excitation current:

$$
\delta \sim \mathrm{Ib} ; \mathrm{P} 2,(\mathrm{Mz}), \mathrm{Un}
$$

If we neglect linearity of waveforms, we could say that the same that holds for torque angle $\delta$ also holds for power factor $(\cos \varphi)$ :

$\cos \varphi \sim \mathrm{Ib} ; \mathrm{P} 2,(\mathrm{Mz}), \mathrm{Un}$

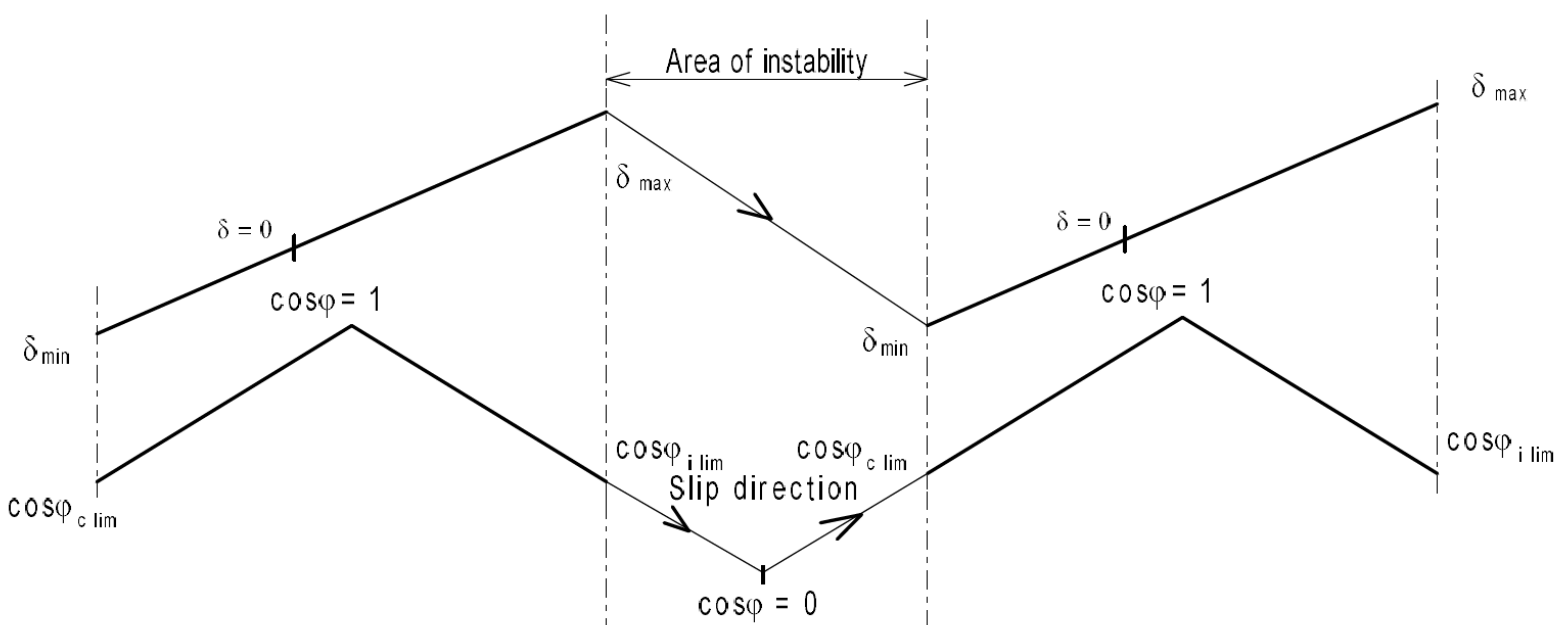

Fig. 4. Comparison of waveforms of torque angle and power factor during slip up. 
The process of slip up in relation to the power factor is shown in Fig. 3.

$\cos \varphi c \lim$ - power factor for $\mathrm{P}=0$, Ibn a Un (idle operation, nominal excitation current), power factor has the lowest value in the capacitive area

$\cos \varphi$ - normal operation power factor, the motor works with nominal load, normal operation value of power factor and nominal stator voltage

$\cos \varphi \mathrm{i}$ lim - power factor that corresponds to the stability limit, has the lowest value in the inductive area. When this value is exceeded the magnetic bond is torn, and the rotor slips up for a pole span.

A comparison of the previously stated bonds between torque angle $\delta$ and power factor $\cos \varphi$ (with neglection of linearity and nonlinearity of both waveforms) is shown in figure 4 .

In figure 5, the same process is shown at the circle diagram.

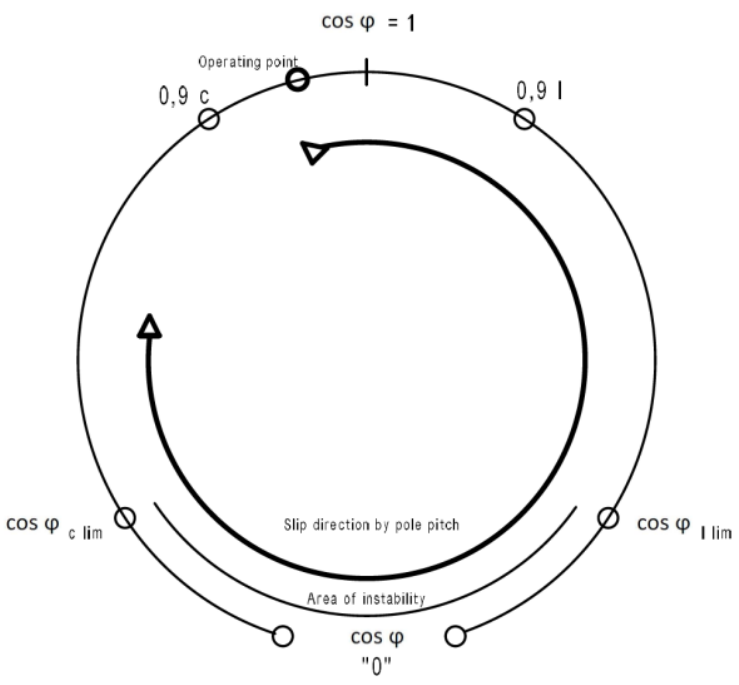

Fig. 5. Circle diagram of a slip up for a pole span.

This diagram corresponds to the view on an analog meter for measurement of power factor with the electromagnetic system without directive torque estimating the power factor from line voltages L1, L2, L3 and phase current. The process of slip up for a pole span shown with bold arrow corresponds to the movement of a pointer of the meter. We can also observe the dynamics of the movement on the pointer where the transit through the unstable part is quick against the transit through the normal working part.

The principle of evaluation of asynchronous operation and design of the appropriate protection for the synchronous machine against this phenomenon is derived from the process described above.

The synchronous machines can be protected against the creation of the asynchronous operation and against the consequences of the asynchronous operation. The former is an operational matter and it is usually one of the functions of the excitation systems. If the machine is getting to the stability limit, we can prevent it by an increase of the stator voltage, lower the load or increase the excitation current. The first two options are very difficult to implement, so the best option is to regulate the excitation current of the machine. This function has many names. It depends on the philosophy of the regulation and the manufacturer. In most cases, it is solved as a "limit of under-excitation," so the parameter is the limit of the power factor.

This parameter can be changed based on the active machine load at advanced regulations, where is the need to use the power and current values of the machine fully. At some machines with low load due to the reluctance torque of the machine (machine with salient poles), the transfer to the synchronous operation will not happen even with zero excitement, so it is not necessary to limit the value of excitation current firmly set by the limit of under excitation.

Under excitation limits also protects the machine against sudden supply voltage drops, short term power shocks, and other transients. Some of the excitation systems can, for some limited time, increase the excitation current to approximately $1.5 \mathrm{Ibn}$ when the machine may fall from synchronism. This short-term increase of excitation current makes it possible to contain the magnetic bond between the stator and the rotor.

\section{Protection AgAinst the CONSEQUENCES OF THE ASYNCHRONOUS OPERATION}

Incorrectly (semantically), they are called "protection against the asynchronous operation." However, these protections do not protect against the creation of asynchronous operation, but they protect only against consequences when it already happens.

These protections are always part of the electric protection of the machine, and their activation means that the drive is shutdown.

Most of these protections work on the impedance principle. Parameter for setting these protections is the reactance of the machine (per unit value of transverse reactance of the machine in excited state Xqsat to the nominal value of reactance of the machine).

The value of Xqsat is hard to obtain. One of the possibilities is prototype measurements and then change the settings based on the tests of the motor with protection.

Based on experiences, the protections, which function is based on the impedance principle (see above), do not work reliably, and they cannot be used for all types of synchronous machines. That is why I later describe the possibility of how to derive the asynchronous operation from the comparisons of the threshold limits power factor.

Based on a general slip up for a pole span that is described above, it is possible to uniquely define the asynchronous operation without the need to know the impedance, power, and construction parameters of the synchronous machine. Protection working on this principle will reliably evaluate asynchronous operation for every type of synchronous and powered machines. 


\section{DESCRIPTION OF PROTECTION AGAINST THE EFFECT OF THE ASYNCHRONOUS OPERATION}

Protection against the effect of asynchronous operation must fulfill these requirements:

a) Reliability of operation

a. The protection must always turn off the machine at the failure events.

b. The protection cannot turn off the machine at normal operation or at transient that does not directly threaten the operation of the drive.

c. The protection must signalize fault warning messages and emergency states.

b) The protection must contain blocking circuits that ensure deactivation of the protection during startup and turning off the drive.

c) It must have a diagnostic of the excitation state of the machine (over-excitement, under excitement, $\cos \varphi=1)$.

d) It must have signalization of operational and faulty states.

a. The protection must signalize fault warning messages and emergency states.

b. It must signalize the actual operating area of the motor (over excited, under excited)

Ad a)

The principle of evaluating a fault state (asynchronous operation, so the slip up for a pole span) must ensure reliable identification of the fault state. It cannot react at the so-called swinging of the rotor (change of torque angle $\delta$ ). On the other side, it must safely evaluate the slip up (loss of pole coherence). Reliability of operation of any component of regulation and control is the basic required attribute.

Ad b)

The synchronous machine is start-up by asynchronous method, so the protection must be disabled during the start until synchronization is completed.

Ad c), d)

The diagnostic of excitation state is suitable for both setting up protection and for maintenance and prophylactics of the protection. It is necessary to realize that the protection as a superior safety part must be regularly controlled, and revision must be done according to valid standards ( ̌́SN in the Czech Republic) and operational regulations.

Also, it is necessary to visualize operational states (presence of voltage, blocking, unblocking, slip up, TRIP). The TRIP function must be signalized even after fault ends (memory with reset).

\section{PROTECTION FUnCTIONALITY}

The functionality principle is derived from machine behavior during slip up for a pole span. The value of power factor change from normal operation (capacitive) power factor through $\cos \varphi=1$ to inductive part and through $\cos$ $\varphi=$ " 0 " (unstable state) go back to the capacitive area during slip up for a pole span (Figure 5). We can say for sure that if the actual power factor changed from inductive area through limit state $\cos \varphi \mathrm{I}$ lim to unstable area $\cos \varphi \mathrm{C}$ $\lim \geq \cos \varphi \mathrm{m} \wedge \cos \varphi \mathrm{m} \leq \cos \varphi \mathrm{I} \lim$ (Figure $5-$ area of instability) and from unstable area through limit state cos $\varphi \mathrm{C} \lim$ to capacitive area that this transient is slip up for a pole span and so it is asynchronous operation. This area is indicated in Figure 5 as an area of instability. The area of instability is an area where the motor cannot operate and gets through it to an over excited state - the area of capacitive $\cos \varphi$.

$\cos \varphi \mathrm{C} \lim$ - border of instability in capacitive state of the machine

$\cos \varphi m$ - operational point - instantaneous power factor of the machine

$\cos \varphi \mathrm{I} \lim$ - border of instability in inductive state of the machine

The limit values $\cos \varphi \mathrm{C} \lim$, and $\cos \varphi \mathrm{I}$ lim cannot be specified generally. They depend on machine construction (cylindrical rotor / salient pole rotor, number of poles, air gap, etc.), nominal values and active load.

Nevertheless, we can say that a magnetic bond is torn at a higher power factor at machines with cylindrical rotors than with salient rotors.

Cylindrical machines can slip up at power factor under 0.9 inductive. Machines with saline rotors have the stability limit at $\cos \varphi$ approx.. under 0.4 inductive.

The exact limits value definition is not so much important. If we use the algorithm described above, the definition of the asynchronous operation is safely defined. We can choose limit values that the machine cannot reach, e.g.:

$\cos \varphi \mathrm{C} \lim =0.4$ cap. [-]

$\cos \varphi \mathrm{I} \lim =0,4$ ind. [-]

Slip up for a pole span alone is not an emergency state for the machine. It is necessary to realize that most of the synchronous machines are constructed for asynchronous start-ups, so it must be designed for short-term asynchronous operation.

Generally, we can say that a limited number of slips in a specified time is not an emergency state for a machine. It is useful to use this fact for the design of machine protection with the possibility to set " $n$ " possible slips in time "T." With this, we eliminate unnecessary emergency shutdowns of drives during transients, short-term voltage drops, shortterm overloads, and so.

\section{CONCLUSION}

Asynchronous operation is a dangerous state of a synchronous machine. During asynchronous operation, the start-up (damping, amortisseur) winding can be damaged a stator winding can get overload by a current that leads to machine destruction.

The protections against asynchronous operation often works incorrectly and unreliable. Either they react in moments that are not dangerous for synchronous machines and cause unnecessary weaning of the drive or they do not respond at real asynchronous operation. It is necessary to set them up complicatedly (often not available parameters are needed), and it is difficult to test and do a revision.

The described detection principle of asynchronous operation clearly defines this state and it is possible to evaluate it reliably. 